\title{
Sensitivity of resistive and Hall measurements to local inhomogeneities
}

\author{
Koon, Daniel W.; Wang, Fei; Petersen, Dirch Hjorth; Hansen, Ole
}

Published in:

Journal of Applied Physics

Link to article, DOI:

$10.1063 / 1.4826490$

Publication date:

2013

Document Version

Publisher's PDF, also known as Version of record

Link back to DTU Orbit

Citation (APA):

Koon, D. W., Wang, F., Petersen, D. H., \& Hansen, O. (2013). Sensitivity of resistive and Hall measurements to local inhomogeneities. Journal of Applied Physics, 114(16), 163710. https://doi.org/10.1063/1.4826490

\section{General rights}

Copyright and moral rights for the publications made accessible in the public portal are retained by the authors and/or other copyright owners and it is a condition of accessing publications that users recognise and abide by the legal requirements associated with these rights.

- Users may download and print one copy of any publication from the public portal for the purpose of private study or research.

- You may not further distribute the material or use it for any profit-making activity or commercial gain

- You may freely distribute the URL identifying the publication in the public portal

If you believe that this document breaches copyright please contact us providing details, and we will remove access to the work immediately and investigate your claim. 


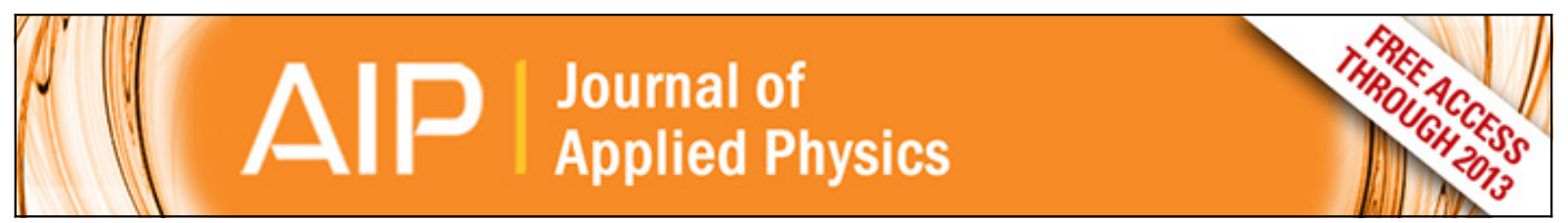

Sensitivity of resistive and Hall measurements to local inhomogeneities

Daniel W. Koon, Fei Wang, Dirch Hjorth Petersen, and Ole Hansen

Citation: Journal of Applied Physics 114, 163710 (2013); doi: 10.1063/1.4826490

View online: http://dx.doi.org/10.1063/1.4826490

View Table of Contents: http://scitation.aip.org/content/aip/journal/jap/114/16?ver=pdfcov

Published by the AIP Publishing

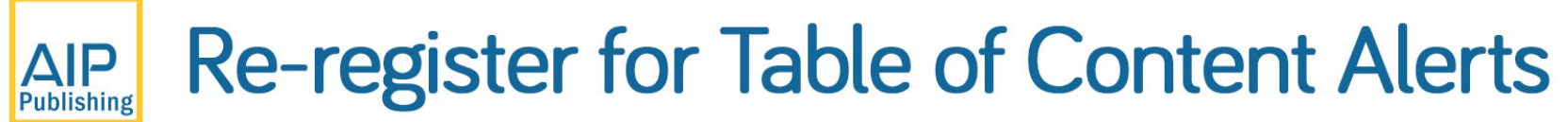

Create a profile.

Sign up today! 


\title{
Sensitivity of resistive and Hall measurements to local inhomogeneities
}

\author{
Daniel W. Koon, ${ }^{1, a)}$ Fei Wang, ${ }^{2,3}$ Dirch Hjorth Petersen, ${ }^{2}$ and Ole Hansen ${ }^{2,4}$ \\ ${ }^{1}$ Physics Department, St. Lawrence University, Canton, New York 13617, USA \\ ${ }^{2}$ Department of Micro- and Nanotechnology, Technical University of Denmark, DTU Nanotech, \\ Building 345 East, DK-2800 Kgs. Lyngby, Denmark \\ ${ }^{3}$ Department of Electronic and Electrical Engineering, South University of Science and Technology of China, \\ Shenzhen, People's of Republic. China 518055 \\ ${ }^{4}$ Danish National Research Foundation's Center for Individual Nanoparticle Functionality (CINF), \\ Technical University of Denmark, DK-2800 Kgs. Lyngby, Denmark
}

(Received 16 July 2013; accepted 27 September 2013; published online 29 October 2013)

\begin{abstract}
We derive exact, analytic expressions for the sensitivity of resistive and Hall measurements to local inhomogeneities in a specimen's material properties in the combined linear limit of a weak perturbation over an infinitesimal area in a small magnetic field. We apply these expressions both to four-point probe measurements on an infinite plane and to symmetric, circular van der Pauw discs, obtaining functions consistent with published results. These new expressions speed up calculation of the sensitivity for a specimen of arbitrary shape to little more than the solution of two Laplace equation boundary-value problems of the order of $N^{3}$ calculations, rather than $N^{2}$ problems of total order $N^{5}$, and in a few cases produces an analytic expression for the sensitivity. These functions provide an intuitive, visual explanation of how, for example, measurements can predict the wrong carrier type in n-type ZnO. (C 2013 AIP Publishing LLC. [http://dx.doi.org/10.1063/1.4826490]
\end{abstract}

\section{INTRODUCTION}

The charge transport measurements, resistivity, $\rho$, and Hall coefficient, $\mathbf{R}_{H}$, are subatomic microscopes, revealing how a material treats its electrons and holes. For a film or thin specimen of thickness, $d$, in a field of magnetic flux density, $B$, measuring one or two four-wire resistances, $R_{i}$, is sufficient for looking through this microscope. These resistances are next converted into the two-dimensional charge transport quantities, sheet resistance, $R_{S}=\rho / d$, and Hall sheet resistance, $R_{H S}=\mathrm{R}_{H} B / d$, before being converted

$$
\begin{aligned}
& R_{i} \rightarrow R_{S} \rightarrow \rho \\
& R_{i} \rightarrow R_{H S} \rightarrow \mathrm{R}_{H} \quad \text { (conversion to } 2 \mathrm{D}, \\
& \quad \text { 3D charge transport quantities) }
\end{aligned}
$$

into the charge transport quantities, using well-known formulas appropriate to the specific measurement technique. ${ }^{1-6}$ However, those formulas break down when the local values of the charge transport quantities vary within the sample. Recent studies of semiconducting $\mathrm{ZnO}$, for example, have confirmed that a highly radially inhomogeneous square specimen can yield the wrong sign for the Hall signal, which might explain some confusion in the literature as to whether the principal charge carriers are electrons or holes. ${ }^{7,8}$ Researchers had used the van der Pauw $[v d P]$ method $^{1,2}$ but had failed to place electrodes at the edges of the films, as required by the vdP technique. The general analytical description of four terminal measurements with electrodes placed in the interior of a film with insulating boundaries has more recently been developed to describe this case., One would expect similar issues with another four-wire

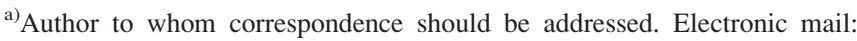
dkoon@stlawu.edu
}

measurement technique, the four-point-probe [4PP] approach, ${ }^{3,4}$ which allows researchers to move an array of four point electrodes throughout the interior of a film, making it ideal for testing the uniformity of semiconductor wafers during micro- or nanofabrication. ${ }^{10}$

The study of the effects of macroscopic inhomogeneity on charge transport properties dates back over sixty years to the study of Hall measurement sensitivity to inhomogeneous magnetic fields, ${ }^{11-17}$ but more recently one group of researchers [SLU group], studying this problem for vdP geometries, defined, numerically calculated, and then directly measured what they have called resistive and Hall weighting functions, $f$ and $g,{ }^{18-23}$ for a variety of specimen shapes, quantifying the sensitivity of charge transport measurement to local inhomogeneities in $R_{S}$ and $R_{H S}$. This group's work showed in a rigorous fashion much of what had already been largely assumed by researchers: the advantages of using square specimens rather than circular ones, ${ }^{18}$ of using cloverleafs and crosses rather than circular and square discs,${ }^{20}$ and of placing electrodes at the corners of a square specimen rather than along its edges. ${ }^{18}$ Another group of researchers [DTU group], studying sheet resistance and Hall (micro-Hall) effect measurements with linear 4PP arrays, developed a complementary notation ${ }^{24-26}$ and has numerically calculated sensitivities of measured configuration resistances, $R_{i, m}$, to local variations of not only $R_{S}$ and $R_{H S}$ but also of the specimen's microscopic materials properties, such as sheet carrier density, $N_{S}$, and mobility, $\mu$.

In this paper, we derive the relation between the SLU and DTU notations and we develop an analytic expression for these weighting functions (or sensitivities), solving several geometries analytically. Along the way we compare these calculated functions to numerical analysis of pathological Hall measurements in semiconducting $\mathrm{ZnO}$, confirming the usefulness of these functions in predicting the effects of macroscopic transport inhomogeneities. 

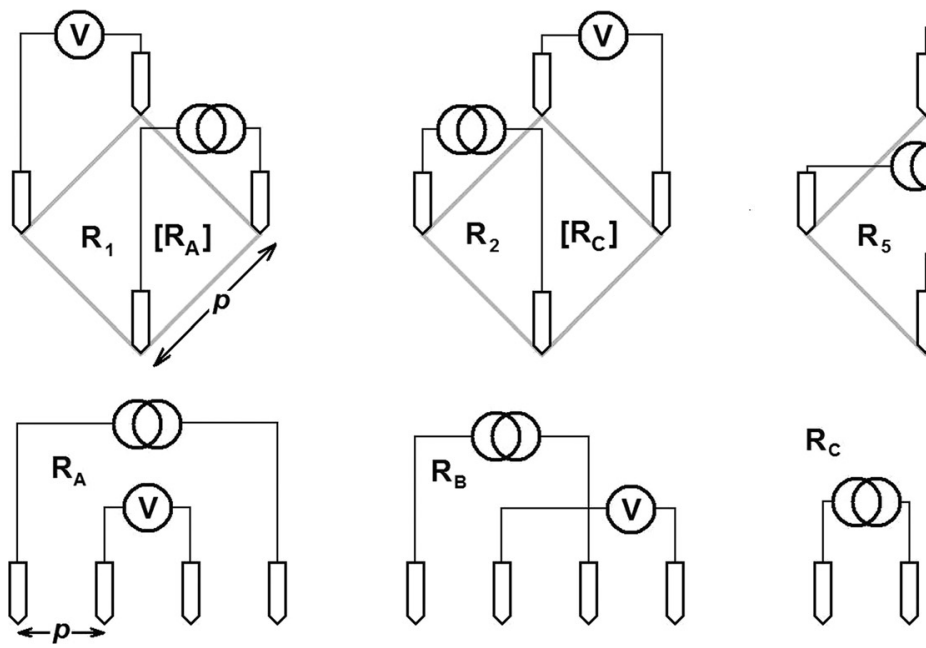

FIG. 1. Principal resistance configurations, $R_{i}$, for $\mathrm{vdP}(\mathrm{i}=1,2,5)$ and square $4 \mathrm{PP}$ geometries $(\mathrm{i}=\mathrm{A}, \mathrm{B}, \mathrm{C})$ (above) and for the linear 4PP geometry (below). The distance between adjacent electrodes, the pitch, $p$, is marked for $R_{1}$ above and $R_{A}$ below.

\section{FOUR-WIRE CHARGE TRANSPORT MEASUREMENT}

Ignoring current reversal, there are six configurations, which we will label by their resistances, $R_{i}$, for attaching a current source to a specimen having four electrodes. Half of these are shown in Figure 1, both for the vdP geometry (above, $i=1,2,5$ ) and for the linear 4PP array (below, $i=\mathrm{A}, \mathrm{B}, \mathrm{C}$ ), while for each of these configurations there is another, $\tilde{R}_{i}$, which we shall call its reciprocal configuration, for which the Reciprocity Theorem states that $\tilde{R}_{i}=R_{i}$, formed by simply exchanging current and voltage electrodes. A pair of reciprocal configurations are shown in Fig. 2 for both a square vdP and a linear 4PP arrangement. Although equal for zero magnetic field, the application of a magnetic field near a vdP or micro-Hall specimen can cause $R_{i}$ and $\tilde{R}_{i}$ to differ by an amount proportional to $R_{H S}$, the Hall sheet resistance.

Sheet resistance measurements are performed via single or dual configuration measurements. In single configuration

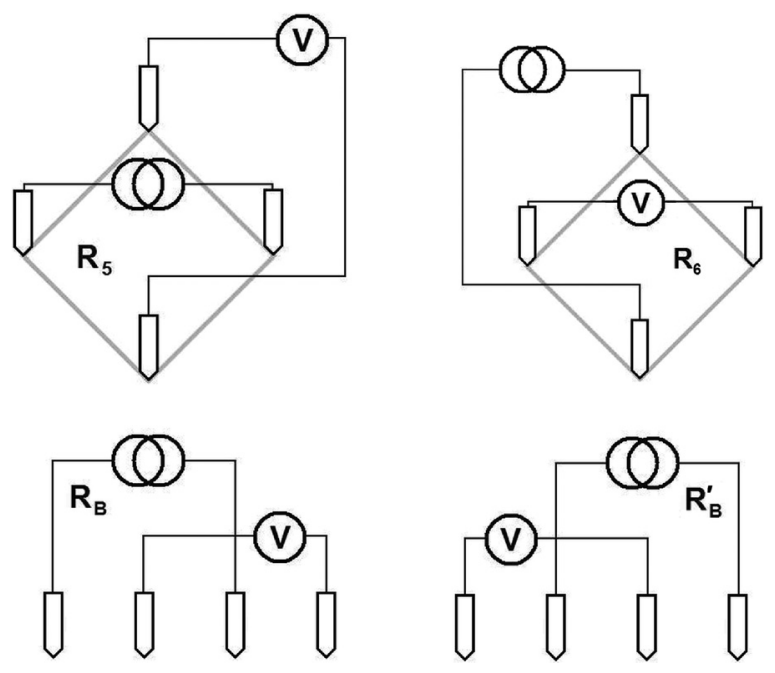

FIG. 2. Two resistance configurations and their reciprocal configurations. Top: the vdP configuration $R_{5}$ and its reciprocal $\tilde{R}_{5}$. Bottom: the linear 4PP configuration $R_{B}$ (left) and its reciprocal $\tilde{R}_{B}$ (right). The remaining reciprocal configurations, $\tilde{R}_{i}$, are also obtained by swapping current electrodes for voltage electrodes. measurements, resistance, $R_{i}$, is measured and converted to sheet resistance, $R_{S, m}$, through the linear relation

$$
R_{S, m}=\alpha_{i} R_{i} \quad \text { single configuration }
$$

The geometry correction factor $\alpha_{i}$ is determined from knowledge of the specimen geometry and electrode positions. Examples of $\alpha_{i}$ are shown in Table I for the limiting cases of equidistant 4PP measurements on an infinite plane and symmetric $\left(R_{1}=R_{2}\right)$ vdP measurements.

Hall measurement is more direct (this does not include micro-Hall which rely on geometrical correction). In the presence of a magnetic field, the van der Pauw geometry ideally allows for the direct calculation of the Hall sheet resistance either by reversing the field or by measuring both $R_{5}$ and its reciprocal configuration

$$
R_{H S}=\left\{\begin{array}{lc}
\frac{1}{2}\left[R_{5}(B)-R_{5}(-B)\right] & \text { reversingfield } \\
\frac{1}{2}\left[R_{5}(B)-\tilde{R}_{5}(B)\right] & \text { single-field }
\end{array}\right.
$$

In this paper we will ignore the zero-field offset in $R_{5}$ and assume only the $B$-dependent portion of $R_{5}$ when we write $R_{5, B}$.

For sheet resistance characterization it is advantageous to perform dual-configuration measurements over singlemeasurement and solving the transcendental equations $1,2,27,28$

TABLE I. Values for the normalization constant, $\alpha_{i}=R_{S} / R_{i}$, for the three interdependent zero-magnetic-field configurations for both 4PP on an infinite plane and symmetric vdP techniques, as defined in Fig. 1. In the absence of a magnetic field, $R_{A}=R_{B}+R_{C}$ and $R_{1}=R_{2}+R_{5}$.

\begin{tabular}{ll}
\hline \hline $\begin{array}{l}\text { Equidistant four-point } \\
\text { probe (4PP) in infinite plane }\end{array}$ & $\begin{array}{l}\text { Symmetric van der } \\
\text { Pauw (vdP) }\end{array}$ \\
\hline$\alpha_{A}=\frac{\pi}{\ln 2}=4.532$ & $\alpha_{1}=\frac{\pi}{\ln 2}=4.532$ \\
$\alpha_{B}=\frac{2 \pi}{\ln 3}=5.719$ & $\alpha_{2}=\frac{\pi}{\ln 2}=4.532$ \\
$\alpha_{C}=\frac{2 \pi}{\ln (4 / 3)}=21.84$ & $\alpha_{5}=$ undefined \\
\hline
\end{tabular}




$$
\begin{array}{ll}
e^{-2 \pi R_{A} / R_{S}}+e^{-2 \pi R_{C} / R_{S}}=1 & \text { linear } 4 P P, \text { infinite plane }, \\
e^{-\pi R_{1} / R_{S}}+e^{-\pi R_{2} / R_{S}}=1 & v d P,
\end{array}
$$

since these can eliminate the need for detailed knowledge of the specimen geometry in calculating $\alpha_{i}$ in Table I. However, while the strict application of the vdP method requires one to measure two separate resistances and solve the above equation to extract $R_{S}$, one may simply measure one four-point resistance for a material of uniform composition and assume a constant $\alpha_{i}$ throughout the measurement, provided there are no variations in material properties, saving time and calculation. One disadvantage of taking this shortcut is that some regions of the specimen are negatively sensitive to local variations in charge transport for a single configuration, implying that the measured value $R_{S, m}$ or $R_{H S, m}$ may fall outside its range of local values within the specimen, as seen for $R_{H S, m}$ in the $\mathrm{ZnO}$ data. ${ }^{7,8}$ This problem vanishes for true vdP dual measurements. ${ }^{18}$ For the remainder of this document we will focus mostly on singleconfiguration measurements since this method is very commonly used in research, due to simplicity.

For nonzero magnetic flux density $B \neq 0$, geometrical magnetoresistance adds an additional correction factor when electrodes are not all placed on specimen boundaries. ${ }^{28}$ For symmetric vdP specimens and $B=0, R_{5}=\tilde{R}_{5}=0$, and so $\alpha_{5}$ is undefined, meaning that one cannot use configuration $i=5$ of a perfectly symmetric vdP specimen for measuring the sheet resistance.

\section{THE TERMINOLOGY OF SENSITIVITY}

The SLU group defines resistive and Hall weighting functions, $f_{i}$ and $g_{i}$ - generalized for any configurations, $R_{i}$ dimensionless quantities satisfying

$$
\begin{aligned}
R_{S, m} & =\alpha_{i} R_{i}=\frac{1}{\Omega} \int_{\Omega} R_{S, L} f_{i} d \Omega^{\prime}, \\
R_{H S, m} & =\frac{1}{\Omega} \int_{\Omega} R_{H S, L} g_{i} d \Omega^{\prime},
\end{aligned}
$$

and

$$
\int_{\Omega} f_{1} d \Omega^{\prime}=\int_{\Omega} f_{2} d \Omega^{\prime}=\int_{\Omega} g_{5} d \Omega^{\prime}=\Omega
$$

where $R_{S, L}\left[R_{H S, L}\right]$ is the local value of the [Hall] sheet resistance, and the integration proceeds over the area of the specimen, $\Omega$. The first of these expressions can be written as a second derivative, as the DTU group has noted ${ }^{24,25}$

$$
f_{i}=\Omega \alpha_{i} \frac{\partial^{2} R_{i, m}}{\partial A \partial R_{S, L}}=\Omega \alpha_{i} \lim _{\Delta R_{S, L} / R_{S} \ll 1} \frac{\Delta R_{i, m}}{\Delta A \Delta R_{S, L}}[\mathrm{vdP}],
$$

where $\alpha_{i}=R_{S} / R_{i}$ (Table I), $\Delta A$ is the area of a perturbation in the local sheet resistance of size $\Delta R_{S, L}$. The derivative form of the equation is appropriate for the weak, small-area limit $\left(\Delta R_{S, L} / R_{S} \ll 1, \Delta A / A \ll 1\right)$, and the finite difference form is more appropriate to stronger or larger-area inhomogeneities. The generalized dimensionless sensitivity ${ }^{24,25}$ has the form

$$
S_{t}^{T}=\frac{p^{2} \Delta T / T}{\Delta A(\Delta t / t)}
$$

in which $p$ is the electrode pitch (Fig. 1) and the perturbation of a local property $t$ (e.g., $R_{S, L}$ or $R_{H S, L}$ ) alters some macroscopic property, $T$. In this formalism, the DTU group defines resistive sensitivities as

$$
S_{R_{S, L}}^{R_{i, m}}=p^{2} \alpha_{i} \frac{\partial^{2} R_{i, m}}{\partial A \partial R_{S, L}}=p^{2} \alpha_{i} \lim _{\Delta R_{S, L} / R_{S} \ll 1} \frac{\Delta R_{i, m}}{\Delta A \Delta R_{S, L}}[4 \mathrm{PP}] .
$$

From this we see that the SLU and DTU formalisms are equivalent, except for the choice of effective normalization area, $A$, with $A=\Omega$ for $\mathrm{vdP}$ and $A=p^{2}$ for the 4PP technique, or

$$
\left.\begin{array}{c}
f_{i} \\
S_{R_{S, L}}^{R_{i, m}}
\end{array}\right\}=A \alpha_{i} \frac{\partial^{2} R_{i, m}}{\partial A \partial R_{S, L}}=A \alpha_{i} \lim _{\Delta R_{S, L} / R_{S} \ll 1} \frac{\Delta R_{i, m}}{\Delta A \Delta R_{S, L}} \quad\left\{\begin{array}{l}
v d P \\
4 P P
\end{array} .\right.
$$

The sensitivity to the local Hall sheet resistance can be written as

$$
\left.\begin{array}{c}
g_{i} \\
S_{R_{H S, L}}^{R_{H S, m}}
\end{array}\right\}=A \frac{\partial^{2} R_{H S, m}}{\partial A \partial R_{H S, L}}=A \lim _{\Delta R_{H S, L} / R_{H S} \ll 1} \frac{\Delta R_{H S, m}}{\Delta A \Delta R_{H S, L}} \quad\left\{\begin{array}{l}
v d P \\
4 P P
\end{array} .\right.
$$

\section{THE ZERO-FIELD, INFINITESIMAL-AREA, WEAK LIMIT}

The sheet current density, $\mathbf{J}_{S}=\mathbf{J} \cdot d$, is related to the electric field by $\mathbf{J}_{S}=\mathbf{G} \mathbf{E}$, where the elements of the conductance tensor are given by $\mathbf{G}=\left(\begin{array}{cc}G_{d} & G_{h} \\ -G_{h} & G_{d}\end{array}\right)$, and the direct and Hall conductances, $G_{d}$ and $G_{h}$, are related in turn both to $R_{S}$ and $R_{H S}$, and the basic materials properties by

$$
\begin{aligned}
G_{d} & =\frac{R_{S}}{R_{S}^{2}+R_{H S}^{2}}=\frac{\cos ^{2} \Theta_{H}}{R_{S}}=\frac{N_{S} e \mu}{1+\mu^{2} B^{2}}, \\
G_{h} & =\frac{R_{H S}}{R_{S}^{2}+R_{H S}^{2}}=\frac{\sin 2 \Theta_{H}}{2 R_{S}}=\frac{r_{H} N_{S} e \mu^{2} B}{1+\mu^{2} B^{2}}=\mu_{H} B G_{d},
\end{aligned}
$$

where $N_{S}$ is the sheet carrier density, $\mu$ is the carrier mobility (and $\mu_{H}$ is the Hall mobility), and $B$ is the magnetic flux density. By convention, we take $\mathbf{B}=B \mathbf{e}_{z}$ normal to the sample surface. The Hall angle is defined by $\tan \Theta_{H}=R_{H S} / R_{S}$ $=G_{h} / G_{d}$, and the Hall scattering factor, $r_{H}$, is of order 1 and varies weakly with $\mu, B$, and the volume carrier density, $n=N_{S} / d$. We will use the approximation $\mu_{H} \approx \mu$ for the remainder of this paper.

For a thin laminar sheet in the xy-plane with a magnetic field along the positive $\mathrm{Z}$-axis, the continuity equation $\nabla \cdot \mathbf{J}_{S}=0$ (except at current injection points) implies 


$$
\begin{aligned}
\nabla^{2} \phi= & -\frac{I}{G_{d}}\left[\delta\left(\mathbf{r}-\mathbf{r}_{+}\right)-\delta\left(\mathbf{r}-\mathbf{r}_{-}\right)\right] \\
& +\frac{\nabla G_{d}}{G_{d}} \cdot \mathbf{E}+\mathbf{e}_{\mathbf{z}} \cdot \frac{\nabla G_{h}}{G_{d}} \times \mathbf{E}, \\
= & -\frac{I}{G_{d}}\left[\delta\left(\mathbf{r}-\mathbf{r}_{+}\right)-\delta\left(\mathbf{r}-\mathbf{r}_{-}\right)\right]-\frac{\nabla \cdot \mathbf{G}}{G_{d}} \cdot \nabla \phi,
\end{aligned}
$$

for a current source and sink at $\mathbf{r}_{+}$and $\mathbf{r}_{-}$, respectively. A local perturbation $G_{d} \rightarrow G_{d}+\Delta G_{d}$ and $G_{h} \rightarrow G_{h}+\Delta G_{h}$ inside a region of area $\Delta A$ around a point $\mathbf{r}_{0}$ simplifies, in the $\Delta A \rightarrow 0$ limit, to

$$
\begin{aligned}
G_{d} & \rightarrow G_{d}+\Delta A \Delta G_{d} \delta\left(\mathbf{r}-\mathbf{r}_{0}\right), \\
G_{h} & \rightarrow G_{h}+\Delta A \Delta G_{h} \delta\left(\mathbf{r}-\mathbf{r}_{0}\right),
\end{aligned}
$$

where the effective normalization area is $A=\Omega\left[A=p^{2}\right]$ for the vdP [4PP] specimen and, as noted in Refs. 18 and 19, a delta-like local perturbation in $G_{d}\left[G_{h}\right]$ produces an effect identical to placing a point dipole at that location, $\mathbf{r}_{0}$, proportional to and parallel [perpendicular] to the local electric field, $\mathbf{E}\left(\mathbf{r}_{0}\right)$.

In earlier works ${ }^{18-23}$ the SLU group has referred to the sensitivity functions calculated from Eq. (3) as the "resistivity weighting function" and the "Hall weighting function," stating that the latter was due to inhomogeneities in the Hall angle. Equation (3) suggests that we should instead call $-f_{i}$ the "conductance weighting function" and $g_{i}$ the "Hall conductance weighting function," since $G_{d}$ and $G_{h}$ are the quantities that are averaged by the measurement process, not $R_{S}$ and $R_{H S}$.

In the linear limit of weak perturbations in $G_{d}$ and $G_{h}$ over an infinitesimal area, the local electric field is unchanged by the perturbation and we can express it in terms of the Green's function

$$
\mathbf{E}(\mathbf{r})=\frac{I}{G_{d}}\left[\nabla \mathrm{G}\left(\mathbf{r}, \mathbf{r}_{+}\right)-\nabla \mathrm{G}\left(\mathbf{r}, \mathbf{r}_{-}\right)\right] .
$$

Meanwhile, we can use Green's formula

$$
\begin{aligned}
\phi(\mathbf{r})= & \int_{\Omega} \mathrm{G}\left(\mathbf{r}, \mathbf{r}^{\prime}\right) \nabla^{2} \phi\left(\mathbf{r}^{\prime}\right) d \Omega^{\prime} \\
& +\int_{\omega}\left[\phi\left(\mathbf{r}^{\prime}\right) \nabla^{\prime} \mathrm{G}\left(\mathbf{r}, \mathbf{r}^{\prime}\right)-\mathrm{G}\left(\mathbf{r}, \mathbf{r}^{\prime}\right) \nabla^{\prime} \phi\left(\mathbf{r}^{\prime}\right)\right] d \omega^{\prime},
\end{aligned}
$$

where $\nabla^{2} \mathbf{G}\left(\mathbf{r}, \mathbf{r}_{0}\right)=\delta\left(\mathbf{r}-\mathbf{r}_{0}\right)$ and $\omega$ is the specimen boundary, to calculate $\phi$ from Eq. (3). For a specimen of infinite area, or in the $B=0$ case, we can ignore the second integral. For now we consider the $\mu B \ll 0$ case. Plugging Eq. (3) into Green's formula produces

$$
\begin{aligned}
\phi(\mathbf{r})= & -\frac{I}{G_{d}}\left[\mathrm{G}\left(\mathbf{r}, \mathbf{r}_{+}\right)-\mathrm{G}\left(\mathbf{r}, \mathbf{r}_{-}\right)\right]+\frac{I \Delta A \Delta G_{d}}{G_{d}^{2}} \int_{\Omega} \mathrm{G}\left(\mathbf{r}, \mathbf{r}^{\prime}\right) \nabla^{\prime} \delta\left(\mathbf{r}^{\prime}-\mathbf{r}_{0}\right) \cdot\left[\nabla^{\prime} \mathrm{G}\left(\mathbf{r}^{\prime}, \mathbf{r}_{+}\right)-\nabla^{\prime} \mathrm{G}\left(\mathbf{r}^{\prime}, \mathbf{r}_{-}\right)\right] d \Omega^{\prime} \\
& +\frac{I \Delta A \Delta G_{h}}{G_{d}^{2}} \mathbf{e}_{\mathbf{z}} \cdot \int_{\Omega} \mathrm{G}\left(\mathbf{r}, \mathbf{r}^{\prime}\right) \nabla^{\prime} \delta\left(\mathbf{r}^{\prime}-\mathbf{r}_{0}\right) \times\left[\nabla^{\prime} \mathrm{G}\left(\mathbf{r}^{\prime}, \mathbf{r}_{+}\right)-\nabla^{\prime} \mathrm{G}\left(\mathbf{r}^{\prime}, \mathbf{r}_{-}\right)\right] d \Omega^{\prime} \\
= & -\frac{I}{G_{d}}\left[\mathrm{G}\left(\mathbf{r}, \mathbf{r}_{+}\right)-\mathrm{G}\left(\mathbf{r}, \mathbf{r}_{-}\right)\right]-\left.\left.\frac{I \Delta A \Delta G_{d}}{G_{d}^{2}} \nabla^{\prime} \mathrm{G}\left(\mathbf{r}, \mathbf{r}^{\prime}\right)\right|_{\mathbf{r}^{\prime}=\mathbf{r}_{0}} \cdot\left[\nabla \mathrm{G}\left(\mathbf{r}, \mathbf{r}_{+}\right)-\nabla \mathrm{G}\left(\mathbf{r}, \mathbf{r}_{-}\right)\right]\right|_{\mathbf{r}=\mathbf{r}_{0}} \\
& -\left.\frac{I \Delta A \Delta G_{h}}{G_{d}^{2}} \mathbf{e}_{\mathbf{z}} \cdot \nabla^{\prime} \mathrm{G}\left(\mathbf{r}, \mathbf{r}^{\prime}\right)\right|_{\mathbf{r}^{\prime}=\mathbf{r}_{0}} \times\left.\left[\nabla \mathrm{G}\left(\mathbf{r}, \mathbf{r}_{+}\right)-\nabla \mathrm{G}\left(\mathbf{r}, \mathbf{r}_{-}\right)\right]\right|_{\mathbf{r}=\mathbf{r}_{0}} .
\end{aligned}
$$

If we measure the voltage across the voltage probes, $\tilde{\mathbf{r}}_{+}$and $\tilde{\mathbf{r}}_{-}$, which are also the current probes for the reciprocal configuration, $\tilde{R}_{i}$, we find that the perturbed resistance, $R_{i}+\Delta R_{i}$ due to a point perturbation at $\mathbf{r}_{\mathbf{0}}$ is

$$
\begin{aligned}
R_{i, m}+\Delta R_{i, m}= & \frac{\phi\left(\tilde{\mathbf{r}}_{+}\right)-\phi\left(\tilde{\mathbf{r}}_{-}\right)}{I} \\
\Delta R_{i, m}= & \left.\left.\frac{\Delta A \Delta G_{d}}{G_{d}^{2}}\left[\nabla \mathrm{G}\left(\mathbf{r}, \tilde{\mathbf{r}}_{+}\right)-\nabla \mathrm{G}\left(\mathbf{r}, \tilde{\mathbf{r}}_{-}\right)\right]\right|_{\mathbf{r}=\mathbf{r}_{0}} \cdot\left[\nabla \mathrm{G}\left(\mathbf{r}, \mathbf{r}_{+}\right)-\nabla \mathrm{G}\left(\mathbf{r}, \mathbf{r}_{-}\right)\right]\right|_{\mathbf{r}=\mathbf{r}_{0}} \\
& +\left.\frac{\Delta A \Delta G_{h}}{G_{d}^{2}}\left[\nabla \mathrm{G}\left(\mathbf{r}, \tilde{\mathbf{r}}_{+}\right)-\nabla \mathrm{G}\left(\mathbf{r}, \tilde{\mathbf{r}}_{-}\right)\right]\right|_{\mathbf{r}=\mathbf{r}_{0}} \times\left.\left[\nabla \mathrm{G}\left(\mathbf{r}, \mathbf{r}_{+}\right)-\nabla \mathrm{G}\left(\mathbf{r}, \mathbf{r}_{-}\right)\right]\right|_{\mathbf{r}=\mathbf{r}_{0}} \cdot \mathbf{e}_{\mathbf{z}},
\end{aligned}
$$

where we have used the relation $\nabla \mathrm{G}\left(\mathbf{r}^{\prime}, \mathbf{r}\right)=-\nabla \mathrm{G}\left(\mathbf{r}, \mathbf{r}^{\prime}\right)$, etc. So, in the small-perturbation, $B=0$ limit, the resistive weighting function is simply

$$
\left.\begin{array}{c}
f_{i}(\mathbf{r}) \\
S_{R_{S, L}}^{R_{i, m}}
\end{array}\right\}=\alpha_{i} A \frac{\partial^{2} R_{i, m}}{\partial R_{S, L} \partial A}=\alpha_{i} A F_{i}(\mathbf{r}),
$$

where we define

$$
F_{i}(\mathbf{r}) \equiv\left[\nabla \mathrm{G}\left(\mathbf{r}, \mathbf{r}_{+}\right)-\nabla \mathrm{G}\left(\mathbf{r}, \mathbf{r}_{-}\right)\right] \cdot\left[\nabla \mathrm{G}\left(\mathbf{r}, \tilde{\mathbf{r}}_{+}\right)-\nabla \mathrm{G}\left(\mathbf{r}, \tilde{\mathbf{r}}_{-}\right)\right] .
$$

Now, as long as the expected value of $R_{i}$ is not equal to zero, that is, as long as $\alpha_{i}$ is defined, we can normalize this expression in the following form: 


$$
\left.\begin{array}{c}
f_{i}(\mathbf{r}) \\
S_{R_{S, L}}^{R_{i, m}}
\end{array}\right\}=A \frac{F_{i}}{\int F_{i} d \boldsymbol{\Omega}^{\prime}}=A \frac{\mathbf{J}_{S, i} \cdot \tilde{\mathbf{J}}_{S, i}}{\int \mathbf{J}_{S, i} \cdot \tilde{\mathbf{J}}_{S, i} d \Omega^{\prime}}=A \frac{\mathbf{E}_{i} \cdot \tilde{\mathbf{E}}_{i}}{\int \mathbf{E}_{i} \cdot \tilde{\mathbf{E}}_{i} d \mathbf{\Omega}^{\prime}},
$$

in the limit $G_{h} / G_{d} \ll 1$, where $\tilde{\mathbf{J}}_{S}$ and $\tilde{\mathbf{E}}_{i}$ represent the sheet current density and electric field at a point $\mathbf{r}$ for the reciprocal configuration $\tilde{R}_{i}$ and where we have used the normalization to eliminate $\alpha_{i}$. Similarly, the Hall weighting function is

$$
\left.\begin{array}{l}
g_{i}(\mathbf{r}) \\
S_{R_{H S, L}}^{R_{i, m}}
\end{array}\right\}=A \frac{\partial^{2} R_{i, m}}{\partial R_{H S, L} \partial A}=\frac{A}{R_{S}} \frac{\partial^{2} R_{i, m}}{\partial\left(G_{h} / G_{d}\right) \partial A}=A G_{i}(\mathbf{r}),
$$

where we define

$$
\begin{aligned}
G_{i}(\mathbf{r})= & {\left[\nabla \mathrm{G}\left(\mathbf{r}, \mathbf{r}_{+}\right)-\nabla \mathrm{G}\left(\mathbf{r}, \mathbf{r}_{-}\right)\right] } \\
& \times\left[\nabla \mathrm{G}\left(\mathbf{r}, \tilde{\mathbf{r}}_{+}\right)-\nabla \mathrm{G}\left(\mathbf{r}, \tilde{\mathbf{r}}_{-}\right)\right] \cdot \mathbf{e}_{\mathbf{z}} .
\end{aligned}
$$

This expression is also easy to normalize provided that the configuration $R_{i}$ is a Hall configuration for a van der Pauw geometry, that is, that its B-field-dependent component equals the Hall sheet resistance, $R_{H S}$

$$
\begin{aligned}
& \left.\begin{array}{l}
g_{i}(\mathbf{r}) \\
S_{R_{H S, L}}^{R_{i, m}}
\end{array}\right\}=\frac{A G_{i}}{\int G_{i} d \boldsymbol{\Omega}^{\prime}}=\frac{A\left(\mathbf{J}_{S, i} \times \tilde{\mathbf{J}}_{S, i}\right) \cdot \mathbf{e}_{\mathbf{z}}}{\int\left(\mathbf{J}_{S, i} \times \tilde{\mathbf{J}}_{S, i}\right) \cdot \mathbf{e}_{\mathbf{z}} d \boldsymbol{\Omega}^{\prime}} \\
& =\frac{A\left(\mathbf{E}_{i} \times \tilde{\mathbf{E}}_{i}\right) \cdot \mathbf{e}_{\mathbf{z}}}{\int\left(\mathbf{E}_{i} \times \tilde{\mathbf{E}}_{i}\right) \cdot \mathbf{e}_{\mathbf{z}} d \mathbf{\Omega}^{\prime}}
\end{aligned}
$$

(special case of van der Pauw Hall configuration).

The normalizations of Eqs. (4b) and (5b) simplify the task of solving for these weighting functions for arbitrary or nonsymmetric geometries. All that is required to calculate the sensitivity functions is to solve two Laplace equation boundary value problems: once for the configuration of interest and once for its reciprocal configuration. If these two problems are solved numerically on say an $N \times N$ grid with a numerical technique that converges with order $N$, this is much faster than solving a similar Laplacian for every point on the grid for which we wish to know the sensitivity-a boundary value problem in which we tweak the conductivity at that point-with a time of order $N^{3}$ vs $N^{5}$. It also removes the question of how strong a perturbation is needed in the calculation to avoid noise problems on the one hand and nonlinear effects on the other.

However, we can calculate the sensitivities not only to local sheet resistance and sheet Hall resistance, but to local variations in fundamental materials properties, such as the sheet carrier concentration and the mobility. To compare the various materials sensitivities in this weak-perturbation, small-field limit, we first observe that

$$
S_{t}^{R_{i, m}}=\lim _{\Delta t / t \rightarrow 0} \frac{\left(\Delta R_{i, m} / R_{i, m}\right)}{(\Delta A / A)(\Delta t / t)}=S_{G_{d}}^{R_{i, m}} \frac{\partial G_{d}}{\partial t} \frac{t}{G_{d}}+S_{G_{h}}^{R_{i}} \frac{\partial G_{h}}{\partial t} \frac{t}{G_{h}},
$$

from which we obtain

$$
\begin{aligned}
& S_{N_{S}}^{R_{i, m}}=\alpha_{i} A\left(-F_{i}+\mu B G_{i}\right) \\
& S_{\mu}^{R_{i, m}}=\alpha_{i} A\left(-F_{i}+2 \mu B G_{i}\right) \\
& S_{B}^{R_{i, m}}=\alpha_{i} A \mu B\left(G_{i}+2 \mu B F_{i}\right),
\end{aligned}
$$

in the limit of $\Delta t / t \ll 1, \mu B \ll 1$. We must be cautious in applying Eq. (6) because the quantities $F_{i}$ and $G_{i}$ themselves have nonzero $B$-field dependence. The problem of nonzero magnetic fields will be considered in another article. ${ }^{29}$

While we have derived Eqs. (4) and (5) for the case of a conducting plane of infinite area, the equations appear to be of general validity for all simply connected specimens, as calculating the functions on the right-hand side and comparing them to previously calculated (and experimentally measured $^{21,22}$ ) weighting functions for a both circular and square $\mathrm{vdP}^{18-20}$ and both linear and square $4 \mathrm{PP}^{18,24,25}$ geometries confirms. We have included two of these cases, a square vdP arrangement and both a linear and square 4PP array on an infinite conducting plane, in Figs. 3 through 5.

In Fig. 3 we have calculated the weighting function $f_{1}=S_{S, L}^{R_{1, m}}=f_{3}$ for a single-configuration measurement, the "dual" weighting function $\left(f_{1}+f_{2}\right) / 2$, and the Hall weighting function, $g_{5}=g_{6}$ by numerically solving for the electric potential on a $101 \times 101$ grid using a finite difference approach on a Microsoft Excel spreadsheet incorporating Eq. (4) for a square vdP specimen with electrodes at the corners. The values at the center of each figure are 3.2114, 3.2114, and 1.4365 , vs. the values of $3.1573,3.1573$, and 1.3932, respectively, calculated in Appendix A, with discrepancies arising from truncation of the infinite series expression for the electric potential. Color contours for all weighting functions are spaced 0.2 apart along the z-axis.

\section{A. Specific exact expressions}

While the electric potential of the square vdP problem in Fig. 3 cannot be expressed in simple, closed form, there
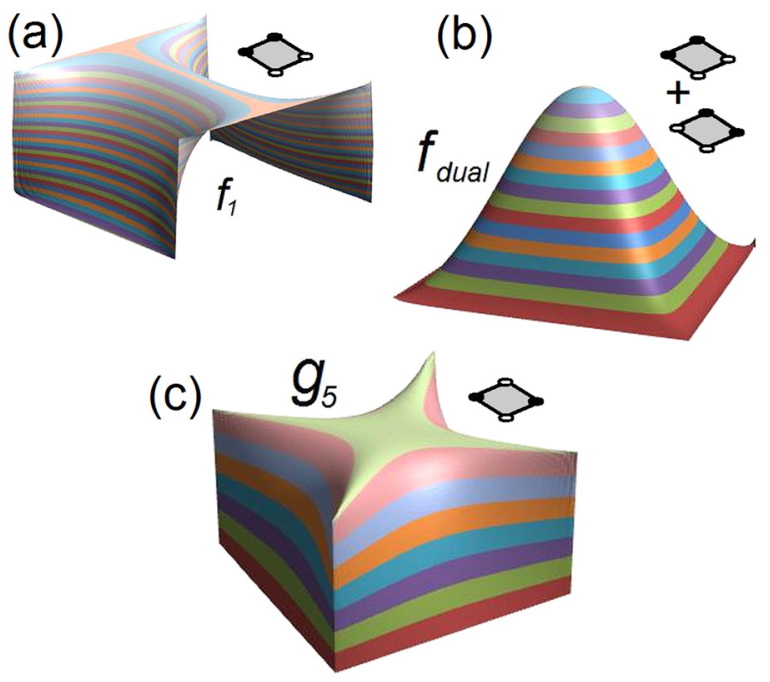

FIG. 3. Weighting functions calculated from Eqs. (4) and (5) for a square $\mathrm{vdP}$ specimen with infinitesimal electrodes at its corners (inset). (a) Resistivity single configuration: $f_{1}=S_{R_{S L}}^{R_{1, m}}$; (b) resistivity dual configuration: $\left(f_{1}+f_{2}\right) / 2$; (c) Hall effect: $g_{5}$. Insets show the corresponding electrode arrangement, with colored pads denoting current probes and white pads denoting voltage probes. 
(a)

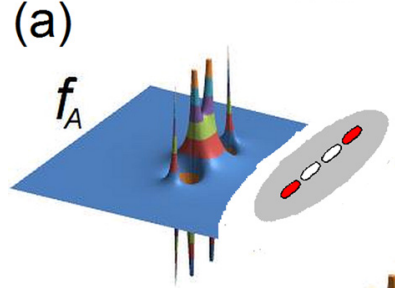

(b)

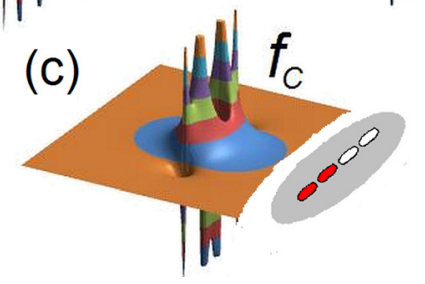

FIG. 4. Sensitivities calculated exactly from Eq. (8), or by solving Eq. (4) for a linear 4PP array on an infinite plane. (a) Resistivity single configuration, $S_{R_{S, L}}^{R_{A}}$, (b) resistivity single configuration, $S_{R_{S, L}}^{R_{B}}$, (c) resistivity single configuration $S_{R_{S L}}^{R_{C}}$. Insets show the corresponding electrode arrangement on the infinite plane, with colored pads denoting current probes and white pads denoting voltage probes. Singularities at the electrodes have been truncated in the graph.

are geometries for which Eqs. (4) and (5) can be expressed in closed algebraic form. This is possible for at least two common vdP geometries - the semi-infinite conducting plane and the circular disc-as well as for all 4PP geometries in the limit of an infinite or semi-infinite conducting plane.

For a vdP circular disc of radius $a$ for which configuration $R_{i}$ is defined by current probes located at $(r, \theta)=(a, \pi)$ and $(a, \pi / 2)$ and voltage probes at $(a, 0)$ and $(a,-\pi / 2)$ (a)
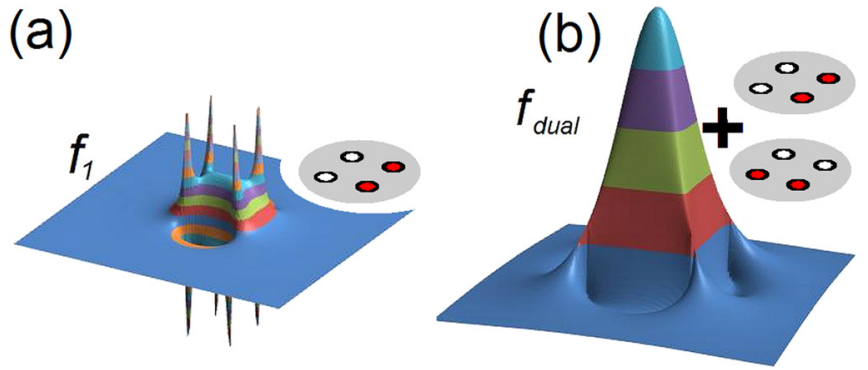

FIG. 5. Sensitivities calculated from Eq. (9), or by solving Eqs. (4) and (5) exactly for a perfectly symmetric square $4 \mathrm{PP}$ array with pitch $p=1$ on an infinite disc. (a) Resistivity: single configuration, $S_{R_{S L}}^{R_{1, m}}$, as defined in Fig. 1; (b) resistivity: dual configuration, $\left(S_{R_{S, L}}^{R_{1, m}}+S_{R_{S, L}}^{R_{2, m}}\right) / 2$, assuming perfect symmetry, $R_{1}=R_{2}$. Insets show the corresponding electrode arrangement, with colored pads denoting current probes and white pads denoting voltage probes.

$$
\begin{aligned}
f_{1} & =\frac{2 a^{4}}{\ln 2} \cdot \frac{\left(r^{2}-a^{2}\right)^{2}-2 a^{2} r^{2} \sin 2 \theta}{r^{8}-2 a^{4} r^{4} \cos 4 \theta+a^{8}} \\
f_{\text {dual }} & =\frac{2 a^{4}}{\ln 2} \cdot \frac{\left(r^{2}-a^{2}\right)^{2}}{r^{8}-2 a^{4} r^{4} \cos 4 \theta+a^{8}} \\
g_{5} & =\frac{4 a^{4}}{\pi} \cdot \frac{a^{4}-r^{4}}{r^{8}-2 a^{4} r^{4} \cos 4 \theta+a^{8}},
\end{aligned}
$$$$
v d P \text { circular disc. }
$$

Cartestian-coordinate versions of this and the following two equations are given in Appendix B. For the general case of four electrodes in an infinite plane

$$
S_{R_{S, L}}^{R_{i, m}}=p^{4} \frac{\left\{\begin{array}{r}
{\left[\left(x-x_{1}\right)\left|\mathbf{r}-\mathbf{r}_{4}\right|^{2}-\left(x-x_{4}\right)\left|\mathbf{r}-\mathbf{r}_{1}\right|^{2}\right] \cdot\left[\left(x-x_{2}\right)\left|\mathbf{r}-\mathbf{r}_{3}\right|^{2}-\left(x-x_{3}\right)\left|\mathbf{r}-\mathbf{r}_{2}\right|^{2}\right]} \\
+\left[\left(y-y_{1}\right)\left|\mathbf{r}-\mathbf{r}_{4}\right|^{2}-\left(y-y_{4}\right)\left|\mathbf{r}-\mathbf{r}_{1}\right|^{2}\right] \cdot\left[\left(y-y_{2}\right)\left|\mathbf{r}-\mathbf{r}_{3}\right|^{2}-\left(y-y_{3}\right)\left|\mathbf{r}-\mathbf{r}_{2}\right|^{2}\right]
\end{array}\right\}}{2 \pi\left|\mathbf{r}-\mathbf{r}_{1}\right|^{2}\left|\mathbf{r}-\mathbf{r}_{2}\right|^{2}\left|\mathbf{r}-\mathbf{r}_{3}\right|^{2}\left|\mathbf{r}-\mathbf{r}_{4}\right|^{2} \ln \left\{\left[\left|\mathbf{r}_{3}-\mathbf{r}_{1}\right|\left|\mathbf{r}_{4}-\mathbf{r}_{2}\right|\right] /\left[\left|\mathbf{r}_{2}-\mathbf{r}_{1}\right|\left|\mathbf{r}_{4}-\mathbf{r}_{3}\right|\right]\right\}},
$$

which reduces to the following for a linear 4PP array of pitch $p$ centered along the $\mathrm{x}$-axis:

$$
\begin{aligned}
& S_{R_{S, L}}^{R_{A, m}}=\frac{3 p^{4}}{2 \pi \ln 4} \cdot \frac{r^{4}-\frac{5}{2} p^{2} r^{2} \cos 2 \theta+\frac{9}{16} p^{4}}{\mathrm{D}(r, p, \theta)}, \\
& S_{R_{S, L}}^{R_{B, m}}=\frac{2 p^{4}}{\pi \ln 3} \cdot \frac{r^{4}-\frac{5}{2} p^{2} r^{2} \cos ^{2} \theta+\frac{1}{2} p^{2} r^{2} \sin ^{2} \theta+\frac{9}{16} p^{4}}{\mathrm{D}(r, p, \theta)}, \\
& S_{R_{S, L}}^{R_{C, m}}= \frac{-p^{4}}{2 \pi \ln \frac{4}{3}} \cdot \frac{r^{4}-\frac{5}{2} p^{2} r^{2} \cos ^{2} \theta-\frac{11}{2} p^{2} r^{2} \sin ^{2} \theta+\frac{9}{16} p^{4}}{\mathrm{D}(r, p, \theta)}, \\
&\left(S_{R_{S, L}}^{R_{S, m}}\right)_{\text {dual }}= \frac{12 p^{4}}{\pi(4 \ln 4-3 \ln 3)} \cdot \frac{p^{2} r^{2} \sin ^{2} \theta}{\mathrm{D}(r, p, \theta)}, \\
& \mathrm{D}(r, p, \theta)= {\left[r^{4}-\frac{9}{2} p^{2} r^{2} \cos 2 \theta+\frac{81}{16} p^{4}\right]\left[r^{4}-\frac{1}{2} p^{2} r^{2} \cos 2 \theta+\frac{1}{16} p^{4}\right] } \\
& \text { where } \quad \text { inear } 4 P P \text { array on infinite conducting plane, }
\end{aligned}
$$

with $\alpha_{A}=\pi / \ln 2, \alpha_{B}=2 \pi / \ln 3$, and $\alpha_{C}=2 \pi / \ln \frac{4}{3}$ (Table I) for the functions of Eq. (8), in excellent agreement with the results of Eqs. (4) and (5) calculated from the finite-difference method (Fig. 4) and those previously published elsewhere. ${ }^{18,24,25}$ 
In Fig. 4 we calculated sensitivities of 4PP resistive measurements for the infinite conducting plane for linear 4PP arrays (Fig. 4). The values calculated from Eq. (8) match the published numerical results at the center in 4PP array in (a) through (c), with $0.6123,1.0302$, and -0.9835 , respectively, agreeing to at least 4 decimal places in each case. Color contours for all weighting functions are spaced 0.2 apart along the z-axis for (a) through (c).

For the square 4PP array with electrodes placed at $(r, \theta)=(p / \sqrt{2}, \pm \pi / 4)$ and $(p / \sqrt{2}, \pm 3 \pi / 4)$,

$$
\begin{aligned}
S_{R_{S, L}}^{R_{A, m}} & =\frac{p^{4}}{4} \cdot \frac{r^{4}-2 p^{2} r^{2} \cos ^{2} \theta+\frac{p^{4}}{4}}{r^{8}+\frac{1}{2} p^{4} r^{4} \cos 4 \theta+\frac{1}{16} p^{8}}, \\
S_{R_{S, L}}^{R_{C, m}} & =\frac{p^{4}}{4} \cdot \frac{r^{4}-2 p^{2} r^{2} \sin ^{2} \theta+\frac{p^{4}}{4}}{r^{8}+\frac{1}{2} p^{4} r^{4} \cos 4 \theta+\frac{1}{16} p^{8}}, \\
\left(S_{R_{S, L}}^{R_{S, m}}\right)_{\text {dual }} & =\frac{p^{4}}{4} \cdot \frac{\left(r^{2}-\frac{p^{2}}{2}\right)^{2}}{r^{8}+\frac{1}{2} p^{4} r^{4} \cos 4 \theta+\frac{1}{16} p^{8}}
\end{aligned}
$$

square 4PP array on infinite conducting plane,

the first and last of which we plot in Fig. 5.

The value at the center of both Figs. 5(a) and 5(b) is 1, regardless of $p$, and the function integrates over area to $p^{2}$. Singularities at the electrodes have been truncated in graph (a). Color contours for all weighting functions are spaced 0.2 apart along the z-axis. The value of both functions at the center of the array is 1 , regardless of pitch.

\section{B. Experimental consequences}

Ideally, these weighting functions for charge transport measurements should be nonnegative functions, unlike the single-configuration resistive weighting function for Figs. 4(a)-4(c) and 5(a) because, in theory, a function for which $f_{i}<0$ or $g_{i}<0$ in some regions of the specimen could lead to charge transport measurements that lie outside the range of values occurring within the specimen itself. This effect has apparently already been observed for n-type $\mathrm{ZnO}$ films in which the Hall signal can have the wrong sign, leading to a misassignment of charge transport polarity. ${ }^{7,8}$ Ohgaki et al. prove that this can occur when there is an internal hole in the specimen, if the electrodes are placed close to that hole rather than at the outer edges of the specimen by numerically solving the perturbed boundary value problem. ${ }^{7}$ Bierwagen et al. show numerically that both radial inhomogeneities and edge inhomogeneities can also produce this effect if the carrier density is increasing toward the edges of the specimen. ${ }^{8}$ All of these pathological cases require that the electrodes be located well inside the boundaries of the specimen, a fundamental violation of the basic rules for the vdP technique. ${ }^{1,2}$

Fig. 6 shows the Hall weighting function for a square specimen with a square internal hole, as suggested by Ohgaki.

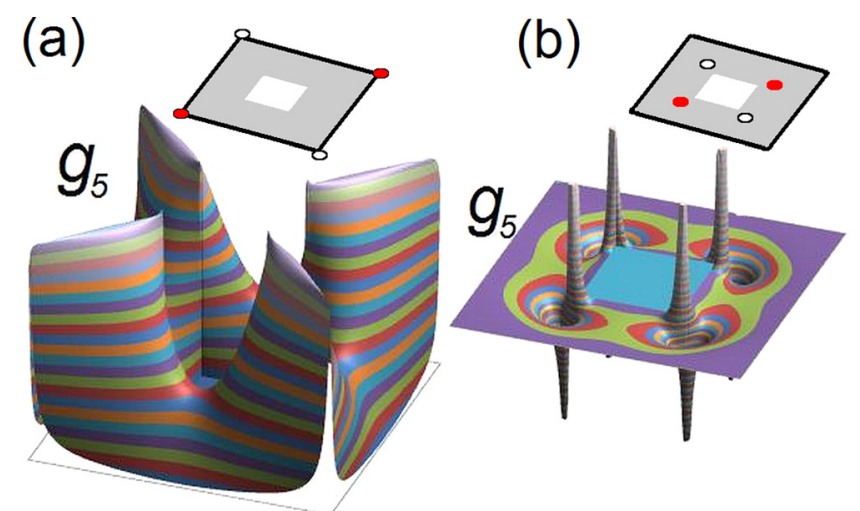

FIG. 6. Hall weighting function, $g_{5}$, for square specimen with square interior hole ( $1 / 3$ the lateral size of the specimen) in the middle, as in Refs. 5 and 6 , with electrodes at the corners (a) and $9 / 10$ of the way in from the corners toward the interior hole (b). The function is positive throughout the specimen in (a), and its average value is negative in (b) so that Hall measurements on a uniform, n-type $\mathrm{ZnO}$ film will yield opposite polarity in the two cases. Color contours for (a) are spaced 0.2 apart along the $\mathrm{z}$-axis. The function in (b) is unnormalized. Insets show the corresponding electrode arrangement, with colored pads denoting current probes and white pads denoting voltage probes.

If the contacts are located at the corners of the specimen, the weighting function is positive throughout, and the measured Hall signal will have the right polarity (Fig. 6(a)). However, if the contacts are not at the corners, singularities develop and, as the electrodes approach the inner hole, the magnitude of the negative contribution to $\int_{\Omega} g_{5} d \Omega$ is greater than the positive contribution. In that case, even a uniform specimen returns the wrong polarity of Hall signal (Fig. 6(b)).

We have also calculated the Hall weighting function, $g_{5}$ in Fig. 7 for a square specimen with electrodes located far inside the boundaries of the specimen both for the homogeneous case and for the case in which the carrier density increases quadratically with distance from the center. This inhomogeneity in $N_{S}$ does two things: first, it changes the shape of $g_{5}=S_{R_{H S L}}^{R_{5}}$ - the magnitude of the negative contribution to $\int_{\Omega} g_{5} d \Omega$ is $99 \%$ of the magnitude of the positive contribution for the inhomogeneous case shown in Fig. 7(b) vs. 70\% for the homogeneous case (Fig. 7(a))—and second it
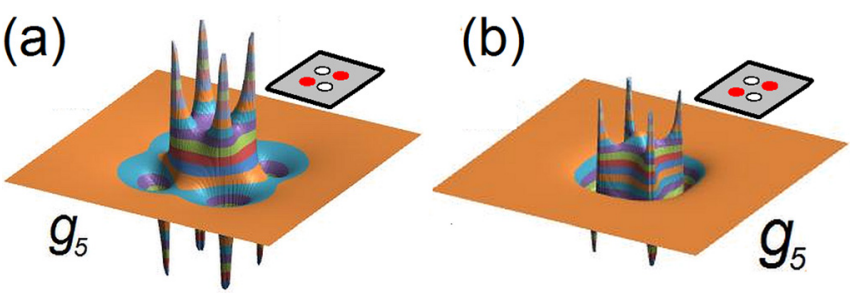

FIG. 7. The Hall weighting function, $g_{5}$, is shown for a square specimen of side $s$ with a distance $0.2 s$ between adjacent electrodes. (a) For specimen of homogeneous carrier density and (b) for specimen in which the density increases radially to 100 times its central value at the corners. The magnitude of the negative contribution to $\int_{\Omega} g_{5} d \Omega$ is $99 \%$ of the positive contribution for the inhomogeneous case shown in (b) vs $70 \%$ for the homogeneous case (a). Radial inhomogeneities in carrier density are shown in Ref. 6 to produce the wrong polarity of Hall signal in this geometry and thus to lead to misinterpretation of carrier type. Both functions are unnormalized. Insets show the corresponding electrode arrangement, with colored pads denoting current probes and white pads denoting voltage probes. 
causes the magnitude of the integral $\int G_{h} g_{5} d \Omega$ $=\int n e \mu^{2} g_{5} d \Omega$ (in the $B=0$ limit) to be larger for regions of negative $g_{5}$ than for regions of positive $g_{5}$, thus producing a negative Hall signal.

\section{CONCLUSIONS}

Sensitivity [weighting] functions provide a powerful, visual, and intuitive tool for interpreting the role of macroscopic inhomogeneities on the charge transport measurement process, allowing one both to quantify the uncertainty in charge transport quantities due to inhomogeneities and to predict when there is a danger that the sort of catastrophic failure already documented for some published $\mathrm{ZnO}$ Hall measurements might occur. We have found a direct expression which makes the process of graphing these sensitivity functions easier for researchers in the laboratory, turning a problem of order $N^{5}$ for a $N \times N$ grid approximation of any arbitrary specimen geometry into a problem of order $N^{3}-$ order $N^{2}$ for a handful of problems that can be solved analytically-allowing the researcher either to greatly reduce the calculation time or to increase the resolution of the function, or both. Further, we have shown that such sensitivity analysis provides a powerful, visual, and intuitive tool for understanding for example how Hall signals can have the wrong sign and for avoiding such pathological cases.

\section{ACKNOWLEDGMENTS}

D.W.K. wishes to thank C. Knickerbocker and B. Watson for helpful collaboration and discussions over the years and to thank P. Bøggild for bringing the authors of the present work together. D.W.K. also wishes to thank The Technical University of Denmark and a St. Lawrence University Research Fellowship for travel support. The Center for Individual Nanoparticle Functionality (CINF) is sponsored by The Danish National Research Foundation (DNRF 54).

\section{APPENDIX A: THE VAN DER PAUW SQUARE AND RELATED GEOMETRIES}

A unit circle, $z$, on the complex plane can be mapped onto the upper half-plane, $u$, and then onto a unit square, $v$, in the complex plane by the following sequence of conformal maps, as illustrated in Fig. 8

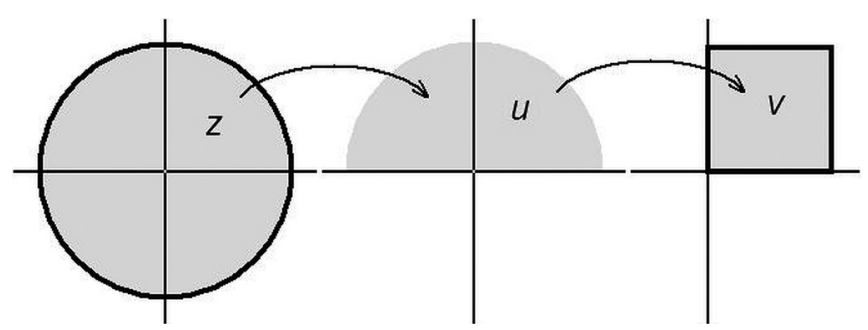

FIG. 8. Conformal mapping of a unit circle onto a unit square in Eq. (A1). The transformation $z \rightarrow v$ can be written in the form of an elliptical integral.

$$
u=+i \frac{1+z}{1-z} \quad v=\frac{1}{k} \int_{0}^{u} \frac{d t}{\sqrt{t\left(1-t^{2}\right)}},
$$

where

$$
\begin{aligned}
k & =\int_{0}^{1} \frac{d t}{\sqrt{t\left(1-t^{2}\right)}}=\int_{1}^{\infty} \frac{2 d t}{\sqrt{t^{4}-1}}=2 \sqrt{\pi} \frac{\Gamma(5 / 4)}{\Gamma(3 / 4)} \\
& \approx 2.62205755 .
\end{aligned}
$$

If we place electrodes symmetrically about the edge of the unit circle at $z=-1,+i,+1$, and $-i$, then these are plotted onto $u=0,-1, \infty$, and +1 on the upper halfplane and $v=0,+i,+1+i$, and +1 , respectively, on the unit square.

Quantities that are conformally invariant under mapping from the circular disc to the square include not only the electric potential for a boundary-value problem on the unit circle, but also the effect of a point perturbation in the conductance within an infinitesimal region of the material, and so both

$$
\frac{\Delta A}{A} f_{i} \text { and } \frac{\Delta A}{A} g_{i}
$$

are conformally invariant. The weighting functions on the square can thus be written as

$$
f_{i}(v)=\frac{k^{2}}{2 \pi}\left|1-z^{4}\right| f_{i}(z) \quad \text { and } \quad g_{i}(v)=\frac{k^{2}}{2 \pi}\left|1-z^{4}\right| g_{i}(z),
$$

since

$$
d u=\frac{2 i d z}{(1-z)^{2}} \quad \text { and } \quad d v=\frac{1}{k} \frac{d u}{\sqrt{u\left(1-u^{2}\right)}}=\frac{\sqrt{2 i}}{k} \frac{d z}{\sqrt{1-z^{4}}} .
$$

While in principle we could use Eq. (A1) to calculate the weighting functions for the square from the analytic expressions on the circle, it is difficult in practice to map an arbitrary point in the interior of the circle onto the square. There are a few points, however, that we can easily map. The center of the circle, for example, maps to the center of the unit square, and so,

$$
\begin{aligned}
& f_{1}(v)=\frac{k^{2}}{\pi \ln 2}=3.157250980, \\
& g_{5}(v)=\frac{2 k^{2}}{\pi^{2}}=1.393203930 \quad(\text { center of square }),
\end{aligned}
$$

both of these values agreeing very well with previously calculated values. ${ }^{18,19}$ We can also map the diagonals (both $y=0$ and $x=0$ ) of the circular disc for the Hall weighting function

$$
g_{5}(z)=\left\{\begin{array}{ll}
4 / \pi\left(1-x^{4}\right) & \text { for } y=0 \\
4 / \pi\left(1-y^{4}\right) & \text { for } x=0
\end{array}\right\}
$$


$g_{5}(v)=\frac{2 k^{2}}{\pi^{2}}=1.393203930$ for both (diagonals of square),

again in excellent agreement with calculated values. Finally, we consider the edges $\left(z=e^{i \phi}\right)$ and the horizontal mirror axis $(y=-x)$ of the circle

$$
f_{1}(z)=\left\{\begin{array}{ll}
\frac{-1}{\ln 2 \cdot \sin 2 \phi} & \text { for } z=e^{i \phi} \\
\frac{2}{\left(1+4 x^{4}\right) \ln 2} & \text { for } y=-x
\end{array}\right\},
$$

$$
f_{1}(v)=\left\{\begin{array}{c}
\frac{k^{2}}{\pi \ln 2} \operatorname{sgn}(\sin 2 \phi)= \pm 3.157250980 \\
\frac{k^{2}}{\pi \ln 2}=3.157250980
\end{array}\right\} \text {, }
$$

$\left(\begin{array}{c}\text { edges, } \\ \text { mirror axis } \\ \text { of square }\end{array}\right)$,

also in excellent agreement with previous calculated values.

\section{APPENDIX B: EXACT FORMS, CARTESIAN COORDINATES}

Equations (7)-(9) for the sensitivities of a circular vdP disc and for both linear and square 4PPs can be transformed into Cartesian coordinates. We include them below since those coordinates may sometimes prove more useful than polar

$$
\begin{aligned}
& f_{1}=\frac{2 a^{4}}{\ln 2} \frac{\left(x^{2}+y^{2}-a^{2}\right)^{2}-4 a^{2} x y}{\left[(x-a)^{2}+y^{2}\right]\left[(x+a)^{2}+y^{2}\right]\left[x^{2}+(y-a)^{2}\right]\left[x^{2}+(y+a)^{2}\right]}, \\
& \frac{1}{2}\left(f_{1}+f_{2}\right)=\frac{2 a^{4}}{\ln 2} \frac{\left(x^{2}+y^{2}-a^{2}\right)^{2}}{\left[(x-a)^{2}+y^{2}\right]\left[(x+a)^{2}+y^{2}\right]\left[x^{2}+(y-a)^{2}\right]\left[x^{2}+(y+a)^{2}\right]}, \\
& g_{5}=\frac{4 a^{4}}{\pi} \frac{a^{4}-\left(x^{2}+y^{2}\right)^{2}}{\left[(x-a)^{2}+y^{2}\right]\left[(x+a)^{2}+y^{2}\right]\left[x^{2}+(y-a)^{2}\right]\left[x^{2}+(y+a)^{2}\right]} \quad \text { Eq. 7(a) (circular vdP disc), } \\
& S_{R_{S, L}}^{R_{A}}=\frac{3 p^{4}}{2 \pi \ln 4} \frac{\left(x^{2}+y^{2}\right)^{2}+\frac{9}{16} p^{4}-\frac{5}{2} p^{2}\left(x^{2}-y^{2}\right)}{\left[\left(x+\frac{3}{2} p\right)^{2}+y^{2}\right]\left[\left(x+\frac{1}{2} p\right)^{2}+y^{2}\right]\left[\left(x-\frac{1}{2} p\right)^{2}+y^{2}\right]\left[\left(x-\frac{3}{2} p\right)^{2}+y^{2}\right]} \\
& S_{R_{S, L}}^{R_{B}}=\frac{2 p^{4}}{\pi \ln 3} \frac{\left(x^{2}+y^{2}\right)^{2}+\frac{9}{16} p^{4}-\frac{5}{2} p^{2} x^{2}+\frac{1}{2} p^{2} y^{2}}{\left[\left(x+\frac{3}{2} p\right)^{2}+y^{2}\right]\left[\left(x+\frac{1}{2} p\right)^{2}+y^{2}\right]\left[\left(x-\frac{1}{2} p\right)^{2}+y^{2}\right]\left[\left(x-\frac{3}{2} p\right)^{2}+y^{2}\right]} \\
& S_{R_{S, L}}^{R_{C}}=\frac{-p^{4}}{2 \pi \ln \frac{4}{3}} \frac{\left(x^{2}+y^{2}\right)^{2}+\frac{9}{16} p^{4}-\frac{5}{2} p^{2} x^{2}-\frac{11}{2} p^{2} y^{2}}{\left[\left(x+\frac{3}{2} p\right)^{2}+y^{2}\right]\left[\left(x+\frac{1}{2} p\right)^{2}+y^{2}\right]\left[\left(x-\frac{1}{2} p\right)^{2}+y^{2}\right]\left[\left(x-\frac{3}{2} p\right)^{2}+y^{2}\right]}
\end{aligned}
$$

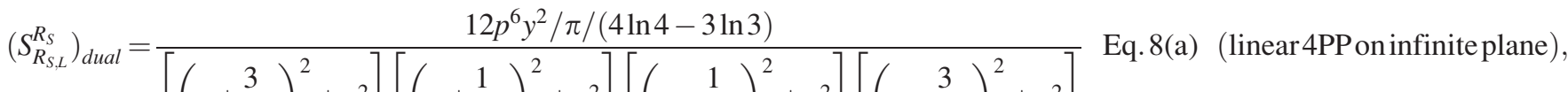

$$
\begin{aligned}
& {\left[\left(x+\frac{3}{2} p\right)^{2}+y^{2}\right]\left[\left(x+\frac{1}{2} p\right)^{2}+y^{2}\right]\left[\left(x-\frac{1}{2} p\right)^{2}+y^{2}\right]\left[\left(x-\frac{3}{2} p\right)^{2}+y^{2}\right]} \\
& S_{R_{S, L}}^{R_{A}}=\frac{p^{4}}{4} \frac{\left(x^{2}+y^{2}\right)^{2}-2 p^{2} x^{2}+\frac{p^{4}}{4}}{\left[\left(x-\frac{p}{2}\right)^{2}+\left(y-\frac{p}{2}\right)^{2}\right]\left[\left(x-\frac{p}{2}\right)^{2}+\left(y+\frac{p}{2}\right)^{2}\right]\left[\left(x+\frac{p}{2}\right)^{2}+\left(y-\frac{p}{2}\right)^{2}\right]\left[\left(x+\frac{p}{2}\right)^{2}+\left(y+\frac{p}{2}\right)^{2}\right]}, \\
& S_{R_{S, L}}^{R_{B}}=\frac{p^{4}}{4} \frac{\left(x^{2}+y^{2}\right)^{2}-2 p^{2} y^{2}+\frac{p^{4}}{4}}{\left[\left(x-\frac{p}{2}\right)^{2}+\left(y-\frac{p}{2}\right)^{2}\right]\left[\left(x-\frac{p}{2}\right)^{2}+\left(y+\frac{p}{2}\right)^{2}\right]\left[\left(x+\frac{p}{2}\right)^{2}+\left(y-\frac{p}{2}\right)^{2}\right]\left[\left(x+\frac{p}{2}\right)^{2}+\left(y+\frac{p}{2}\right)^{2}\right]} \text {, } \\
& \left(S_{R_{S, L}}^{R_{S}}\right)_{\text {dual }}=\frac{p^{4}}{4} \frac{\left(x^{2}+y^{2}-\frac{p^{2}}{2}\right)^{2}}{\left[\left(x-\frac{p}{2}\right)^{2}+\left(y-\frac{p}{2}\right)^{2}\right]\left[\left(x-\frac{p}{2}\right)^{2}+\left(y+\frac{p}{2}\right)^{2}\right]\left[\left(x+\frac{p}{2}\right)^{2}+\left(y-\frac{p}{2}\right)^{2}\right]\left[\left(x+\frac{p}{2}\right)^{2}+\left(y+\frac{p}{2}\right)^{2}\right]}
\end{aligned}
$$

Eq. 9 (a) (square 4PP on infinite plane). 
${ }^{1}$ L. J. van der Pauw, Philips Res. Rep. 13, 1 (1958).

${ }^{2}$ L. J. van der Pauw, Philips Tech. Rev. 20, 220 (1958).

${ }^{3}$ L. B. Valdes, Proc. IRE 42, 420 (1954).

${ }^{4}$ F. M. Smits, Bell Syst. Tech. J. 37, 711 (1958).

${ }^{5}$ D. H. Petersen, O. Hansen, R. Lin, and P. F. Nielsen, J. Appl. Phys. 104, 013710 (2008).

${ }^{6}$ D. H. Petersen, O. Hansen, P. Bøggild, R. Lin, P. F. Nielsen, D. Lin, C. Adelmann, A. Alian, C. Merckling, J. Penaud, G. Brammertz, J. Goossens, W. Vandervorst, and T. Clarysse, J. Vac. Sci. Technol. B 28, C1C41 (2010). ${ }^{7}$ T. Ohgaki, N. Ohashi, S. Sugimura, H. Ryoken, I. Sakaguchi, Y. Adachi, and H. Haneda, J. Mater. Res. 23, 2293 (2008).

${ }^{8}$ O. Bierwagen, T. Ive, C. G. Van de Walle, and J. S. Speck, Appl. Phys. Lett. 93, 242108 (2008)

${ }^{9}$ F. Østerberg et al., J. Appl. Phys. 110, 033707 (2011).

${ }^{10}$ J. R. Ehrstein, M. C. Croarkin, and H. K. Liu, NIST Spec. Publ. 260-131, 126 (2006).

${ }^{11}$ H. Koppe and J. M. Bryan. Can. J. Phys. 29, 274 (1951).

${ }^{12}$ I. Brunner, Solid-State Electron. 1, 172 (1960).

${ }^{13} \mathrm{P}$. Menzel, Arch. Technisch. Messen Messtechnische Praxis (Munich) 32, 2064 (1961).

${ }^{14}$ I. Hlásnik and J. Kokovec, Solid-State Electron. 9, 585 (1966).

${ }^{15}$ R. W. Rendell and S. M. Girvin, Phys. Rev. B 23, 6610 (1981).

${ }^{16}$ S. J. Bending and A. Oral, J. Appl. Phys. 81, 3721 (1997).
${ }^{17}$ S. Liu, H. Guillou, A. D. Kent, G. W. Stupian, and M. Stupian, J. Appl. Phys. 83, 6161 (1998).

${ }^{18}$ D. W. Koon and C. J. Knickerbocker, Rev. Sci. Instrum. 63, 207 (1992).

${ }^{19}$ D. W. Koon and C. J. Knickerbocker, Rev. Sci. Instrum. 64, 510 (1993).

${ }^{20}$ D. W. Koon and C. J. Knickerbocker, Rev. Sci. Instrum. 67, 4282 (1996).

${ }^{21}$ D. W. Koon and W. K. Chan, Rev. Sci. Instrum. 69, 4218 (1998).

${ }^{22}$ J. K. Scherschligt and D. W. Koon, Rev. Sci. Instrum. 71, 587 (2000).

${ }^{23}$ D. W. Koon, Rev. Sci. Instrum. 77, 094703 (2006).

${ }^{24}$ D. H. Petersen, R. Lin, T. M. Hansen, E. Rosseel, W. Vandervorst, C. Markvardsen, D. Kjær, and P. F. Nielsen, J. Vac. Sci. Technol. B 26, 362 (2008).

${ }^{25}$ F. Wang, D. H. Petersen, T. M. Hansen, T. R. Henriksen, and P. Bøggild, J. Vac. Sci. Technol. B 28, C1C34 (2010).

${ }^{26}$ F. Wang, D. H. Petersen, F. W. Osterberg, and O. Hansen, Proceedings of the 17th IEEE International Conference on Advanced Thermal Processing of Semiconductors, RTP 2009 (IEEE, New York, 2009), p. 151.

${ }^{27}$ R. Rymanszewski, J. Phys. E 2, 170 (1969).

${ }^{28}$ S. Thorsteinsson, F. Wang, D. H. Petersen, T. M. Hansen, D. Kjær, R. Lin, J.-Y. Kim, P. F. Nielsen, and O. Hansen, Rev. Sci. Instrum. 80, 053902 (2009).

${ }^{29}$ D. W. Koon, F. Wang, D. H. Petersen, and O. Hansen, "Sensitivity of resistive and Hall measurements to local inhomogeneities. II: Finite-field, intensity, and area corrections" (in preparation). 